\title{
Treatment and Prophylaxis of Invasive Candidiasis with Anidulafungin, Caspofungin and Micafungin - REVIEW OF THE LITERATURE
}

\author{
A. Glöckner \\ BDH Klinik Greifswald GmbH, Greifswald, Germany
}

\begin{abstract}
Working by a distinct cell wall-specific mechanism of action, the echinocandin class of antifungals has substantially expanded the range of available treatments for invasive Candida infections. Anidulafungin, caspofungin and micafungin were investigated versus drugs from earlier antifungal classes in large clinical trials that demonstrated their excellent clinical and microbiological efficacy in the primary treatment of invasive candidiasis. Therefore, and supported by a number of favourable pharmacological characteristics, the echinocandins rapidly became established in guidelines and clinical practice as primary treatment options for moderately to severely ill patients with invasive candidiasis. This article reviews the relevant clinical evidence that forms the basis for the use of echinocandins in the management of invasive candidiasis, and discusses their current role in the context of recent guideline recommendations and treatment optimization strategies.
\end{abstract}

\section{INTRODUCTION}

Candida $s p p$. are prominent fungal pathogens causing invasive infections predominantly in neutropenic and severely ill non-neutropenic patients. Most patients with invasive candidiasis have candidemia without evidence of deep tissue or organ involvement. Despite efforts to advance treatment options and modalities, the mortality associated with invasive candidiasis is still high. Published figures of crude mortality reside in the range of $30-60 \%$, attributable mortality is estimated at 25-40\% [1-6].

As several investigators have shown, mortality of candidemia is directly correlated with the delay of initiation of adequate antifungal therapy $[7,8]$. Thus, early treatment with a reliably active and safe drug is mandatory to achieve the optimum clinical outcomes. However, this is a difficult requirement to meet as the predominant challenges encountered in the management of invasive candidiasis include a lack of well-performing methods for early detection of infection and several shortcomings of the azole and polyene antifungals, the former mainstays of invasive candidiasis therapy. These include gaps in the antifungal spectrum and a multitude of drug interactions for the azoles, and nephrotoxicity or acute toxicity associated with the polyene antifungals.
Beginning in 2002, the introduction of three echinocandin antifungals has significantly expanded the historically limited armamentarium of drugs available for the treatment of invasive candidiasis. Working by a distinct cell wall-specific mechanism of action, anidulafungin, caspofungin and micafungin are characterized by excellent antifungal activity against Candida spp. and Aspergillus spp., low toxicity, few or negligible drug-drug interactions and pharmacokinetic independence of renal (and mostly hepatic) function. Therefore they rapidly became established in guidelines and clinical practice as primary treatment options for severely ill patients with invasive candidiasis. After a brief overview of the epidemiology and characteristics of invasive candidiasis, this article reviews current published data on the use of echinocandins in the management of invasive Candida infections.

\section{INVASIVE CANDIDIASIS}

As pointed out above, invasive Candida infections are important opportunistic infections. In the recent EPIC II survey performed in 667 European intensive care units, Candida spp. were involved in 18.5\% of cases with nosocomial infections [9]. According to another recent survey at the intensive care units of 310 German hospitals [10], fungal pathogens were involved in every fifth patient with infection, in the subset of university hospitals Candida spp. were detected in $24 \%$ of the infections.

In a large survey with 24,000 cases of bloodstream infections in US hospitals [4], Candida spp. were the fourth most common pathogen involved in sepsis at an incidence of 4.6 cases per 10,000 admissions. A multi-institutional survey performed by the European Confederation of Medical Mycology in several European countries reported incidences of 2.0-3.8 cases of candidemia per 10,000 admission and 0.30-0.41 cases per 10,000 patient hospital days. The majority of the cases was diagnosed on surgical and intensive care wards $(48.2 \%$ and $40.2 \%$, respectively), $22.5 \%$ of the patients had solid tumors, $17.4 \%$ received steroids and $12.3 \%$ had hematological malignancies [11].

This distribution reflects the predisposing factors identified by several authors [12], including neutropenia, cancer chemotherapy, colonization with Candida $s p p$., exposure to broad spectrum antibiotics, indwelling central venous catheters, hemodialysis or re- 
nal failure, high APACHE score, mechanical ventilation, prior surgery, particularly gastro-abdominal surgery [13], gastrointestinal perforation and higher age. Another important risk group are solid organ transplant recipients receiving immunosuppressants [14]. Premature birth is a major predisposing factor in neonates [12]. For intensive care patients, the rate of invasive fungal infections increases with the duration of stay, particularly $>7$ days [15].

Among patients with malignant haematological diseases, the highest rates of invasive Candida infections are found in patients with neutropenia due to induction chemotherapy for acute leukemia or myelodysplastic syndrome and in recipients of allogeneic blood stem cell transplants in the early post-transplant phase and in periods of graft-versus-host disease (GVHD) $[16,17]$.

While the majority of invasive Candida infections are blood stream infections, organ involvement after hematogeneous dissemination, peritonitis and endocarditis [18] are important manifestations of disease. Candida endophthalmitis may develop in patients with delayed pathogen clearance from the blood stream (persistent candidemia).

\section{ANTIFUNGAL ACTIVITY OF ECHINOCANDINS IN Clinically Relevant CANDIDA SPP.}

The echinocandins are semisynthetic lipopeptides derived from natural metabolites produced by three different fungi [19]. Their almost identical central hexapeptide structure carries substance-specific lipophilic $\mathrm{N}$-acyl side chains. This may be the basis of some differences in terms of antifungal activity, pharmacokinetics, metabolism, tissue distribution and interactions.

In contrast to azole and polyene antifungals, the echinocandins inhibit the synthesis of 1,3-beta-D-glucan, an essential polysaccharide component of the cell wall in Candida spp. and Aspergillus spp. In Candida $s p p$., the deprivation of this major structural element leads to disruption of the cell wall and subsequent cell lysis, accounting for the fungicidal activity against many Candida isolates at adequate concentrations [20]. The echinocandins show rather similar in vitro antifungal activity with largely overlapping minimal inhibitory concentration ranges for clinically relevant species [21]. MICs against C. albicans, C. glabrata, C. tropicalis and $C$. krusei are usually significantly lower that those observed for $C$. parapsilosis or the rare species C. guilliermondii. However, this divergence apparently does not translate into clearly significant differences in clinical activity. In a recent meta-analysis, the treatment success rate in 202 patients with $C$. parapsilosis infection from five studies was very similar for echinocandins and non-echinocandin drugs (76.3\% vs. $73.0 \%$ ) [22].

Recently proposed modifications of clinical interpretive breakpoints for echinocandin susceptibility testing of Candida spp. reflect this fact: C. parapsilosis breakpoints are at least three dilution steps higher than those of other Candida species, based on the fact that isolates carrying resistance mechanisms are associated with much higher MIC levels in C. parapsilosis vs. other species [23].
Despite increasing use over the last decade, resistance against echinocandins still remains rare and is largely restricted to de novo emergence in patients with longer treatment duration [24]. This is reassuring, given the fact that point mutations in the target enzyme subunit may suffice for resistance induction [25]. Cross-resistance among echinocandins is the rule, while caspofungin-resistant isolates remaining susceptible to anidulafungin and/or micafungin have been observed [26, 27].

\section{Pharmacokinetic Premises}

Due to a lack of oral bioavailability, the echinocandins are exclusively parenteral drugs administered via intravenous infusion over 1-2 hours. Recommended infusion rates should not be exceeded to avoid systemic infusion reaction.

Anidulafungin and micafungin show linear doseplasma exposure relationships while caspofungin exhibits some degree of non-linearity in the clinically useful range [28, 29]. All echinocandins exhibit some degree of enrichment versus plasma in organs relevant to invasive candidiasis (liver, kidneys, spleen, lungs). In animal models, micafungin enriches $2-3$ fold in these organs [30], caspofungin mainly targets the liver and to a lesser extent the kidneys [31] while anidulafungin exhibits rather uniform enrichment (9-12 fold) in all four organs [32]. None of the echinocandins reaches adequate levels in the cerebrospinal fluid, intraocular compartments or urine, making them less suited for treatment of infections involving these sites [33-35].

Anidulafungin is exclusively degraded be spontaneous chemical processes and possibly by nonspecific peptidases in the plasma [36], whereas the other echinocandins are metabolized to some extent in the liver via $\mathrm{N}$-acetylation or by the catechol-O-methyltransferase [37]. This may entail dose adjustment, restrictions of use or drug interactions.

\section{Dosage Considerations}

Anidulafungin is used at a uniform dosage of 100 $\mathrm{mg} / \mathrm{d}$ (preceded by one $200 \mathrm{mg}$ loading dose on day 1) [38] for all patients regardless of body weight, organ functions, age, or comedications [39-41]. Anidulafungin can be used at the same dose in patients with all levels of hepatic insufficiency [40].

Caspofungin dosage must be adjusted according to body weight and liver function. In addition, patients on treatment with inducers of hepatic metabolism must receive higher doses [42]. The standard dosage is $50 \mathrm{mg} / \mathrm{d}$ (after an initial loading dose of $70 \mathrm{mg}$ on day 1). Patients weighing $>80 \mathrm{~kg}$ should receive $70 \mathrm{mg} / \mathrm{d}$ as maintenance dose. Larger doses of caspofungin $(150 \mathrm{mg} / \mathrm{d})$ have been used in a clinical trial on invasive candidiasis. No significant differences or toxicity or efficacy vs. standard doses were noted [43]. Moderate liver insufficiency (Child-Pugh Score 7-9) requires a reduction of the maintenance dose to $35 \mathrm{mg} / \mathrm{d}$ [44]. Caspofungin should not be used in patients with severe hepatic insufficiency (Child-Pugh Score >9) [29].

Micafungin dosage is dependent of the purpose of treatment (therapy vs. prophylaxis) $[45,46]$ and body 
weight [47]. Therapeutic use requires double the dosage compared to prophylactic treatment. In patients weighing $\leq 40 \mathrm{~kg}$ micafungin must be dosed per $\mathrm{kg}$ body weight ( $2 \mathrm{mg} / \mathrm{kg} \mathrm{d}$ or $1 \mathrm{mg} / \mathrm{kg} \mathrm{d}$ for prophylaxis), whereas heavier patients receive a fixed dosage of $100 \mathrm{mg} / \mathrm{d}$ (or $50 \mathrm{mg} / \mathrm{d}$ for prophylaxis). The dosage may be doubled in patients with insufficient initial response.

Micafungin doses are independent of organ functions. However, the drug should not be used in patients with severe liver insufficiency due to a lack of data [47].

\section{DRUG-DRUG INTERACTIONS}

Generally, the echinocandins are characterized by a low potential of drug-drug interactions, a feature that is clearly a novelty in the field of antifungals that notoriously involved multiple pharmacokinetic (azoles) [48] or problematic pharmacodynamic interactions (polyenes) [49].

Primarily owing to the absence of any hepatic metabolism [36], no clinically relevant interactions have been described for anidulafungin [50-52], the drug may thus be used at a fixed dose regardless of any comedications [41].

Due to its hepatic metabolism, caspofungin maintenance dosages should be increased to $70 \mathrm{mg} / \mathrm{d}$ in patients concomitantly receiving enzyme inducers such as rifampicin, dexamethasone, phenytoin, or carbamazepine [42]. Other potential interactions of caspofungin involve immunosuppressants: the comedication of caspofungin and ciclosporin A has been associated with liver function abnormalities in healthy volunteers, therefore patients receiving both drugs should be monitored for liver damage, whereas a retrospective review of patients treated with the combination did not find evidence of enhanced clinically significant hepatotoxicity $[29,53]$. In addition, caspofungin increases the trough levels of tacrolimus [54]; plasma level monitoring and appropriate dose adjustments are required [29].

Micafungin increases the exposure of amphotericin $\mathrm{B}$ requiring risk/benefit analysis and close monitoring of amphotericin B toxicity. In addition micafungin increases the exposures of itraconazole, sirolimus and nifedipine, requiring monitoring and dose reduction as appropriate [47].

\section{Clinical Trials on Treatment of Invasive CANDIDA INFECTIONS}

The discussion in this section is mainly focussed on well-conducted large, prospective trials. Studies with small patient numbers or retrospective trials were not included due to their limited evidence level and ambiguities of clinical interpretation.

\section{Pivotal TRIALS}

The clinical efficacy and safety of all three echinocandins was investigated in randomized double-blinded multicenter trials primarily involving non-neutropenic adult patients with candidemia (Table 1) [45, 55-57].
Two studies used prevalidated comparator regimes anidulafungin was studied versus fluconazole [55] and caspofungin was tested versus conventional amphotericin B [56]. An earlier randomized study had established the therapeutic noninferiority of fluconazole versus amphotericin B [58]. Micafungin, however, was initially investigated vs. liposomal amphotericin B that had not been studied in a randomized trial for this indication [57]. Regulatory authorities therefore requested a second study of micafungin versus caspofungin [45].

All but one study had a primary endpoint of combined clinical-microbiological response at the end of intravenous therapy in the modified intention-to-treat (MITT) population, i.e. patients with initial detection of Candida spp. in blood or other physiologically sterile sites, and receipt of at least 1 dose of study medication. The trial of micafungin vs. liposomal amphotericin B [57] primarily analyzed patients treated for at least 5 days which lead to numerically high success levels in both groups by elimination of patients with early discontinuation or failure. The MITT analysis for non-inferiority was a secondary endpoint in this trial. With the exception of the micafungin vs. liposomal amphotericin B trial, all studies allowed for a switch to oral fluconazole after at least 10 days of intravenous study treatment.

All three trials involving caspofungin and micafungin the non-inferiority of the experimental drug vs. comparator: caspofungin was as effective as amphotericin B and micafungin was non-inferior to liposomal amphotericin B and caspofungin. There were no significant mortality differences between the respective study arms $[45,56,57]$.

The trial of micafungin vs. caspofungin [45] involved two micafungin dose regimens $(100 \mathrm{mg} / \mathrm{d}$ and $150 \mathrm{mg} / \mathrm{d}$ ) that showed similar efficacy, establishing $100 \mathrm{mg} / \mathrm{d}$ as the standard dose in this indication. The global success rates in patients with C. glabrata infections were approximately $20 \%$ higher in the micafungin vs. caspofungin groups, whereas the difference was not statistically significant.

In contrast to the other trials, the protocol of the study comparing caspofungin vs. amphotericin B deoxycholate [56] defined a change of therapy due to toxicity as failure. This caused an inherent imbalance in favour of caspofungin since the well-known nephrotoxicity of amphotericin B necessitated the discontinuation of this study drug in a high proportion of patients $(16.5 \%$ versus $2.8 \%$ in the caspofungin arm).

The trial of anidulafungin versus fluconazole [55] was the first randomized study in invasive candidiasis to establish a significant difference in the primary endpoint favouring a new drug versus an established option. The global success rate at the end of intravenous therapy was $76 \%$ for anidulafungin vs. $60 \%$ for fluconazole. The difference was statistically significant (95\% confidence interval 3.9-27.0). According to the same criteria, anidulafungin had significantly higher success rates at the end of all therapy and at the follow-up visit 2 weeks after the end of therapy. The higher global efficacy of anidulafungin may be due to a faster clearance of the pathogens from the blood 
Table 1. Results in the MITT populations of pivotal trials of echinocandins for therapy of invasive fungal infections [45, 55-57].

\begin{tabular}{|c|c|c|c|c|}
\hline \multirow[b]{2}{*}{ Comparator } & \multirow{2}{*}{$\begin{array}{c}\text { Anidulafungin } \\
\text { Fluconazole }\end{array}$} & \multirow{2}{*}{$\begin{array}{c}\text { Caspofungin } \\
\begin{array}{c}\text { Amphotericin B } \\
\text { deoxycholate }\end{array}\end{array}$} & \multicolumn{2}{|c|}{ Micafungin } \\
\hline & & & $\begin{array}{c}\text { Liposomal } \\
\text { amphotericin B }\end{array}$ & Caspofungin $* * * *$ \\
\hline Patient number (MITT), n & $127 / 118$ & $109 / 115$ & $247 / 247$ & $191 / 188$ \\
\hline Candidemia, $\%$ & $91 / 87$ & $82.6 / 79.1$ & $84.2 / 85.8 * * *$ & $85.3 / 85.6$ \\
\hline Infection with C. non-albicans, $\%$ & $36 / 41 *$ & $\begin{array}{c}64.4 / 45.9 \\
{[p=0.0009]}\end{array}$ & $62.4 / 58.9 * * *$ & $54.5 / 60.6$ \\
\hline Neutropenia, $\%$ & $2.4 / 3.4$ & $12.8 / 8.7$ & $11.9 / 7.9 * * *$ & $11.5 / 5.9$ \\
\hline Switched to oral fluconazole, $\%$ & $26.0 / 28.0$ & $24.8 / 34.8$ & Not allowed & $20.9 / 21.2$ \\
\hline Global success at end of IV therapy, $\%$ & $\begin{array}{c}75.6 / 60.2 \\
{[p=0.01]}\end{array}$ & $\begin{array}{c}73.4 / 61.7 \\
{[\mathrm{p}=\text { n.s. }]}\end{array}$ & $\begin{array}{c}74.1 / 69.6 \\
{[\mathrm{p}=\text { n.s. }]}\end{array}$ & $\begin{array}{c}76.4 / 72.3 \\
{[\mathrm{p}=\text { n.s. }]}\end{array}$ \\
\hline Global success at end of all therapy, $\%$ & $\begin{array}{l}74.0 / 56.8 \\
{[\mathrm{p}<0.02]}\end{array}$ & $\begin{array}{l}72.5 / 61.7 \\
{[\mathrm{p}=\text { n.s. }]}\end{array}$ & $\begin{array}{c}74.1 / 69.6 \\
{[\mathrm{p}=\text { n.s. }]}\end{array}$ & $\begin{array}{l}74.9 / 70.2 \\
{[\mathrm{p}=\text { n.s. }]}\end{array}$ \\
\hline Global success at 2 weeks follow up, $\%$ & $\begin{array}{l}64.6 / 49.2 \\
{[\mathrm{p}<0.02]}\end{array}$ & $\begin{array}{c}63.6 / 53.8 \\
{[\mathrm{p}=\text { n.s. }]}\end{array}$ & Not reported & $\begin{array}{l}54.5 / 50.5 \\
{[\mathrm{p}=\text { n.s. }]}\end{array}$ \\
\hline Global success at 6 weeks follow up, $\%$ & $\begin{array}{l}55.9 / 44.1 \\
{[\mathrm{p}=\text { n.s. }]}\end{array}$ & $\begin{array}{c}56.6 / 47.5^{* *} \\
{[\mathrm{p}=\text { n.s. }]}\end{array}$ & Not reported & $\begin{array}{l}46.6 / 42.6 \\
{[\mathrm{p}=\text { n.s. }]}\end{array}$ \\
\hline $\begin{array}{l}\text { Microbiological success at end of IV } \\
\text { therapy, } \%\end{array}$ & $88.1 / 76.2$ & Not reported & Not reported & $88.5 / 84.0$ \\
\hline Time to negative blood cultures, days & $\begin{array}{c}2 / 5 \\
\text { (C. albicans) }[59]\end{array}$ & Not reported & $3 / 4 * * *$ & $2 / 2$ \\
\hline Persistent infection, $\%$ & $\begin{array}{l}6.3 / 14.4 \\
{[\mathrm{p}=\text { n.s. }]}\end{array}$ & $\begin{array}{l}8.3 / 8.7 \\
{[\mathrm{p}=\text { n.s. }]}\end{array}$ & $\begin{array}{c}8.9 / 8.4 * * * \\
{[\mathrm{p}=\text { n.s. }]}\end{array}$ & $\begin{array}{l}5.8 / 9.6 \\
{[\mathrm{p}=\text { n.s. }]}\end{array}$ \\
\hline Mortality rate (ITT), \% & $\begin{array}{c}22.8 / 31.4 \\
{[\mathrm{p}=\text { n.s. }]}\end{array}$ & $\begin{array}{c}34.2 / 30.4 \\
{[\mathrm{p}=\text { n.s. }]}\end{array}$ & $\begin{array}{c}40 / 40 \\
{[p=\text { n.s. }]}\end{array}$ & $29.0 / 26.4$ \\
\hline
\end{tabular}

* Patients with C. krusei infection were excluded from the trial.

** Follow-up at 6-8 weeks after end of all therapy.

*** In the per-protocol set.

**** Column excludes results of micafungin $150 \mathrm{mg}$ arm.

stream that was obtained e.g. for $C$. albicans after a median of 2 days in patients receiving anidulafungin vs. 5 days in the fluconazole arm [59]. Persistent Candida infection at the end of intravenous therapy was observed in $6.3 \%$ vs. $14.4 \%$ of patients. The robustness of these data was shown by the analysis of subpopulations with risk factors for an unfavourable outcome: anidulafungin achieved higher success rates in intensive care patients, patients with single and multiple organ failure and/or severe sepsis, higher age, and retained central venous catheters [60].

The results of this trial established that anidulafungin - and potentially the echinocandins in general may provide a relevant clinical benefit versus the longstanding standard candidemia drug fluconazole. This advantage may be used to the benefit of more severely ill patients in clinical routine, a notion that is reflected in current guideline recommendations on the treatment of invasive Candida infections.

\section{FURTHER STUDIES}

The database of anidulafungin was recently expanded in a non-comparative multicenter phase IIIb trial (ICE study) [61] exclusively involving adult intensive care patients with invasive Candida infection and at least 1 additional risk factor, e.g. abdominal surgery, organ transplant, or neutropenia. 29\% of the 170 patients had invasive infections involving non-bloodstream body sites. Patients were allowed to switch to oral fluconazole or voriconazole after at least 10 days of intravenous therapy. The success rates at the end of intravenous therapy and further time points were comparable to the results of the pivotal trial, confirming the efficacy of anidulafungin for severely ill patients with invasive candidiasis including those with tissue or organ involvement (Fig. 1).

Another multicenter study investigated the use of caspofungin as primary or salvage treatment in invasive candidiasis involving non-bloodstream body sites. Patients with peritonitis, intraabdominal abscesses, chronic-disseminated candidiasis, or multilocular invasive candidiasis received the standard $50 \mathrm{mg} / \mathrm{d}$ caspofungin dose, patients with osteomyelitis, septic arthritis, or endocarditis were treated with a higher dosage (100 mg/d) [62]. These dosages could be escalated to $100 \mathrm{mg} / \mathrm{d}$ or $150 \mathrm{mg} / \mathrm{d}$, respectively, in cases with inadequate response. The overall success rate was $81 \%$, ranging from $33 \%(1 / 3)$ for endocarditis to $100 \%$ (4/4) for osteomyelitis or septic arthritis. Overall mortality was $23 \%$ at 12 weeks. The elevated dosage of $100 \mathrm{mg} / \mathrm{d}$ was well tolerated.

Colombo et al. [63] analyzed the data of 212 patients with $C$. non-albicans infections (predominantly 
- ICE trial population

- Total anidulafungin arm of pivotal trial

$\square$ Intensive care patients in anidulafungin arm of pivotal trial

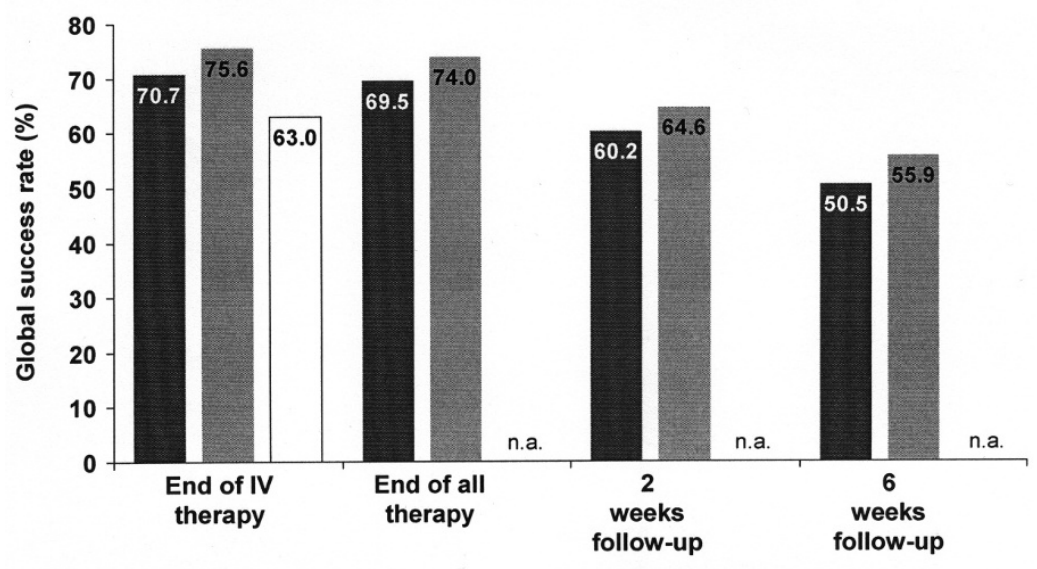

Fig. 1. Global success rates in the anidulafungin ICE trial in intensive care patients and the previous pivotal trial $[55,60,61]$.
C. parapsilosis, C. tropicalis, C. glabrata or C. krusei) from the caspofungin clinical trials database, indicating response rates in the range of infections with $C$. albicans (at least $70 \%$ ).

A recent randomized trial compared caspofungin at elevated dosage $(150 \mathrm{mg} / \mathrm{d})$ versus standard-dose therapy in patients with proven invasive Candida infections, mostly candidemia [43]. Both dosages were equally well tolerated as shown in the primary safety analysis. No significant differences between the study arms were observed in terms of clinical or microbiogical response rates in the secondary efficacy analysis. However, the trial was not sufficiently powered to establish either therapeutic noninferiority or superiority of any treatment arm. A numerically higher response rate of C. parapsilosis infections in the high-dose arm $(17 / 21$ vs. $11 / 18)$ may indicate a benefit in patients with this pathogen species but requires further study due to the lack of statistical significance.

\section{Experience in Neutropenic Patients}

The therapeutic use of echinocandins in neutropenic patients with invasive Candida infection has not been studied in dedicated prospective trials. The echinocandin arms of the pivotal invasive candidiasis trials included comparatively small numbers of patients with neutropenia at baseline (3-50 patients, or 2.4\%-13.3\%) $[45,55-57]$. Keeping the limited database in mind, the pooled response rate for neutropenic patients reported in the trial of micafungin vs. caspofungin was in the same range as in non-neutropenic patients $(68 \%$ vs. $74 \%$ ), without significant differences between the study arms [45]. Micafungin achieved a similar response rate in neutropenic patients as did liposomal amphotericin B $(75 \%$ vs. $80 \%$ in the per protocol set of the trial) [57]. Caspofungin was similarly effective as amphotericin $\mathrm{B}$ deoxycholate in neutropenic patients (50\% vs. $40 \%$ successfully treated patients) [56].

Betts et al. [64] published a compiled analysis of neutropenic patients treated in 4 studies of the caspofungin clinical trials program. Overall success rate in the 27 patients with invasive candidiasis was $68 \%, 15$ of 17 successfully treated patients achieved a complete response.

\section{Deescalation to Azoles}

Based on the results of the pivotal trials of echinocandins that all but one [57] allowed for a switch from intravenous echinocandin to (oral) therapy with fluconazole after 10 days, a step down to an oral azole agent appears feasible after pathogen clearance and clinical stabilization [65]. No detrimental effects of this step down were observed in the approximately $20-35 \%$ of the study patients using this option $[45,55,56]$.

This approach may provide economic benefits and helps to avoid changes of fungal epidemiology and selection of echinocandin resistance via reduced overall exposure to this class of drugs [66].

Prerequisites for a step down to an azole include clinical improvement, resolution of fever, negative blood culture, documented sensitivity of the initial isolate to the intended drug (particularly in patients with C. glabrata infection) and, in the case of oral medication, adequate gastrointestinal function. Pivotal trials that allowed a transition to an oral azole (fluconazole) required 10 days of intravenous therapy $[45,55,56]$. The candidiasis guideline committee of the Infectious Diseases Society of America (IDSA) estimates that 3-5 days of echinocandin therapy followed by oral fluconazole or voriconazole is reasonable while pointing out that few data support this approach [67]. Therefore, the minimum required duration of an initial echinocandin therapy remains uncertain.

\section{Catheter Management}

As biofilms forming on the surfaces of intravenous catheters may serve as a source or reservoir in Candida blood stream infections, current guidelines recommend the removal of catheters in patients with documented candidemia if at all possible [67]. However, as cited by the IDSA guideline panel this recommendation is based primarily on experience in patients receiving antifungals other than echinocandins [67].

Azoles, for that matter, exhibit very little or no useful activity against Candida spp. in biofilms [68, 69]. But as echinocandins have been shown to exert potent antifungal activity against biofilm-dwelling Candida 
cells [68-72], the use of this class of drugs may put the urgency of early catheter removal into perspective. Interestingly, additive effects of neutrophils and anidulafungin on $C$. parapsilosis and $C$. albicans biofilms were recently reported $[73,74]$.

Analyzing data from the two above-mentioned pivotal trials of micafungin vs. caspofungin or liposomal amphotericin B, respectively, Nucci et al. [75] did not find a clinical benefit of early catheter removal. In their multivariate analysis, early catheter removal within 24 or 48 hours of treatment initiation was not associated with higher treatment success or lower mortality. In two of the pivotal trials, response rates of patients with retained catheters were similar to those with catheter removal $[56,57]$. In the study of micafungin vs. caspofungin, patients who underwent catheter removal or replacement at any time had significantly higher response rates than those with remaining catheters in place [45]. This result of an unadjusted analysis may have been influenced by imbalances of the severity of underlying diseases between the two catheter status subgroups. In the trial of anidulafungin vs. fluconazole, the response rates of patients with retained catheter were higher in the anidulafungin group, whereas the number of patients was small (4 vs. 11 patients) [55].

With due caution, it may be concluded that an echinocandin could be preferred over fluconazole as initial therapy for patients in whom early catheter removal appears to be unfeasible or associated with inappropriate risks or complications. Deescalation to an azole appears inadequate in these patients.

However, it should be noted that the guideline panel of the European Conference on Infections in Leukemia (ECIL-3) strongly recommends catheter removal in all infections involving $C$. parapsilosis probably because this species is particularly prone to biofilm formation and generally less susceptible to echinocandins vs. other species [76].

\section{Experience in Pediatric Patients With INVASIVE CANDID $A$ INFECTIONS}

Limited available data on the use or echinocandins for treatment of invasive Candida infections in pediatric patients include prospective clinical trials for caspofungin and micafungin. Both agents are licensed for the treatment of invasive candidiasis in children $(>12$ months of age for caspofungin).

Caspofungin was studied in a multicenter trial that involved 37 patients (age: 3 months to 17 years) with invasive Candida infections, mostly candidemia [77]. In 30 patients with primary therapy, complete responses were observed in $81 \%$ of cases. Five of 7 patients receiving caspofungin for salvage therapy were successfully treated. The response rates were largely independent of age, but the small sample size does not allow valid conclusions regarding this aspect. Two out of five neutropenic patients were successfully treated. In 4 patients, the dosage was escalated from 50 to 70 $\mathrm{mg} / \mathrm{m}^{2}$ due to inadequate treatment response, with successful outcome in 3 of them.

The efficacy of micafungin in pediatric patients with invasive candidiasis was investigated in a subpop- ulation of the above-mentioned double-blind, randomized trial. This substudy [78] compared micafungin (2 $\mathrm{mg} / \mathrm{kg}$ ) with liposomal amphotericin B (3 $\mathrm{mg} / \mathrm{kg}$ ) as first-line therapy of invasive candidiasis in 106 children and adolescents $<16$ years of age; $57 \mathrm{pa}-$ tients were $\leq 2$ years old, 19 patients were premature infants. Successful outcomes were observed in $73 \%$ of patients of the micafungin group versus $76 \%$ of those treated with liposomal amphotericin B. Treatment success was independent of neutropenia or prematurity status. Adverse events leading to discontinuation of study drug were significantly less frequent in the micafungin group.

Pediatric patients aged $\geq 6$ months were included in a randomized trial investigating prophylaxis of invasive fungal infections in recipients of blood stem cell transplants [46]. A total of 84 pediatric patients $(<16$ years), mostly receiving allogeneic hematopoietic stem cell transplantation (HSCT) were analyzed. Proven or probable breakthrough infections occurred in 1 of 39 pediatric patients in the micafungin group (no Candida infections) and 3 of 45 in the fluconazole group (including 1 candidemia).

Published clinical experience with anidulafungin in children is limited to date [79]. Clinical trial data of anidulafungin in pediatric patients with invasive candidiasis have not been presented yet; a non-comparative trial enrolling 60 patients with invasive candidiasis is ongoing [80].

Echinocandins, i.e. currently caspofungin and micafungin, provide valuable and well-tolerated treatment options for pediatric patients with Candida infections.

\section{Prophylaxis of InVASIVE CANDIDIASIS}

Prophylactic use of systemic antifungals is an established practice in hematological and selected nonhematological patients at high risk of invasive candidiasis or other invasive fungal infections [81, 82]. Several studies have explored the use of echinocandins in this indication.

Clinical experience in the prophylactic treatment with caspofungin includes hematological patients and liver transplant recipients. Mattiuzzi et al. [83] performed an open-label randomized comparison of caspofungin versus itraconazole in 192 patients receiving chemotherapy for malignant haematological diseases. Seven patients in the caspofungin arm developed invasive fungal infections (including 2 with candidemia), compared to 5 in the itraconazole arm (including 4 with candidemia). The limited size of the trial precluded definitive conclusions about the relative prophylactic efficacy of both drugs. Chou et al. [84] reported on a retrospective analysis of 123 blood stem cell recipients (117 with allogeneic HSCT; 50 of whom developed GVHD) who had received caspofungin $(35-50 \mathrm{mg} / \mathrm{d})$ over a median duration of 73 days for prophylaxis of invasive fungal disease. Nine patients $(7.3 \%)$ developed breakthrough invasive fungal infections including 2 with Candida infections). The authors concluded that caspofungin appears to be an effective option for primary antifungal prophylaxis in the highly immunosuppressed stem cell transplant patient population. Fortun et al. [85] described a 
prospective trial in 71 liver transplant recipients receiving prophylactic caspofungin for at least 21 days, the observation period spanned 100 days. Two patients developed breakthrough fungal infection including one $C$. albicans surgical wound infection.

Micafungin was investigated in a large phase III randomized double blind trial versus fluconazole for prophylaxis in 882 allogeneic HSCT recipients of all ages during the neutropenic phase for up to 42 days. Seven breakthrough infections occurred in the micafungin arm (including 4 candidemias) versus 11 in the fluconazole group (including 2 candidemias) [46]. Based on the results of this trial, micafungin was licensed for prophylaxis of invasive Candida infections in allogeneic HSCT patients.

The prophylactic use of anidulafungin has not been investigated as yet.

Thus, randomized trials on prophylaxis with echinocandins indicate their potential usefulness in the prevention of Candida infection in hematological high risk patients. However, study data in non-hematological patients are insufficient to support prophylactic treatment with echinocandins in non-study settings for this population. In addition, their routine prophylactic use would expose high numbers of patients to these agents for prolonged periods of time with potential untoward consequences, e.g. selection of resistance in the exposed patients, and shifts in the local epidemiology towards less echinocandin-susceptible strains. Given the restricted options for the treatment of patients after exposure to echinocandins and/or selection of low-susceptibility strains, particularly $C$. glabrata and C. krusei, the widespread use of these agents appears unjustified, let alone the burden of intravenous application and high cost.

\section{SAFETY AND TOLERABILITY}

The echinocandins have generally favourable safety and tolerability profile (Table 2). Most observed adverse events (AEs) are mild to moderate in nature. The most frequent adverse events include infusion reactions (predominantly phlebitis and fever), liver enzyme abnormalities, mild hypokalemia, gastrointestinal symptoms, skin rash and headache [29, 41, 91].

Infusion reactions such as fever or rigors that may occur more often with caspofungin versus the other echinocandins [86] may be managed by reducing the rate of infusion. Phlebitis may be avoided by infusion via a central venous catheter [54] after early replacement in the course of therapy. Liver enzyme abnormalities are generally mild and were mostly less common or similarly frequent as in the comparator groups of randomized trials [55-57].

Echinocandins are rated as pregnancy category C drugs and should not be used in pregnant women [8789]. As these drugs may be secreted in the milk, breastfeeding should be avoided.

In the pivotal trial of anidulafungin vs. fluconazole, the total adverse event rates were similar in both arms [55]. Anidulafungin was associated with significantly lower incidence of hepatic enzyme abnormalities $(1.5 \%$ vs. $7.2 \%$ of patients; $\mathrm{p}=0.03)$. Two patients experienced treatment-related serious AEs in the anidulafungin arm (1 patient with atrial fibrillation, 1 with seizures). The most frequent treatment related AEs (all grades) were hypokalemia (3.1\%), diarrhea (3.1\%) and elevated ALT levels (2.3\%). There was 1 treatment discontinuation $(<1 \%)$ due to an adverse event [90].

As expected, caspofungin was significantly better tolerated than amphotericin B deoxycholate in the pivotal invasive candidiasis trial [56]. Drug-related infusion reactions, laboratory abnormalities and nephrotoxic events were significantly less frequent in the caspofungin group: $20 \%$ (vs. $49 \%$ ) of patients had an infusion-related event (mostly chills, fever or phlebitis/thrombophlebitis), 11\% (vs. 26\%) developed hypokalemia requiring supplementation, $24 \%$ (vs. $54 \%$ ) developed laboratory abnormalities (mostly liver function test changes) and $8 \%$ (vs. 25\%) had a nephrotoxic effect. Three percent (vs. 23\%) discontinued study treatment due to adverse events.

Micafungin showed a generally similar safety and tolerability profile versus liposomal amphotericin B in the first phase III trial in invasive candidiasis [57]. 5\% (vs. $9 \%$ ) of patients discontinued therapy due to an

Table 2. Frequencies of drug-related adverse events observed in patients receiving echinocandins [86].

\begin{tabular}{lccc}
\hline Adverse reaction, \% of patients & Anidulafungin & Caspofungin & Micafungin \\
\hline Phlebitis & $<1$ & $3.5-25$ & 1.6 \\
Fever & $<1$ & $4-40$ & $1-14$ \\
Abdominal pain & $<2$ & 3.6 & 1 \\
Nausea / vomiting & $1 /<1$ & $1-6 / 2-4$ & $2-7 / 1-5$ \\
Diarrhea & 3.1 & 3.6 & 1.6 \\
Headache & 1.3 & $4-15$ & $2-17$ \\
Rash / pruritus & $1 /<2$ & $1-10 /<2$ & $1-12 /<1$ \\
Leukopenia & $<1$ & 6.2 & 1.6 \\
Neutropenia & 1 & 1.9 & 1.2 \\
Thrombocytopenia & $<2$ & 3.1 & $<1$ \\
Hypokalemia & $3-10$ & $2-10$ & 1.2 \\
Liver function test abnormalities & $3-5$ & $1-15$ & $1-8$ \\
\hline
\end{tabular}


adverse event. The most frequent micafungin-related AEs were infusion related reactions (17\%), fever (8\%), hypokalemia $(7 \%)$ and nausea $(5 \%)$. Drug-related laboratory abnormalities mostly included moderate liver function test abnormalities (2-7\%). The second pivotal trial comparing micafungin vs. caspofungin reported similar overall drug-related AE profiles in the two micafungin and the caspofungin arms [45]. The types of adverse events were not reported per treatment group in the publication. Adverse events in the total population predominantly included liver function test abnormalities, nausea, constipation, hypokalemia and rash; $2-4 \%$ of patients withdrew from the trial due to adverse events.

As a unique aspect or micafungin, foci of altered hepatocytes and hepatocellular tumours were observed after prolonged exposure in preclinical animal experiments, with a threshold for tumour induction in the range of human therapeutic exposure [91]. While the clinical relevance of these findings remains unclear, the European Medicines Agency restricted the use of micafungin to situations where other antifungals are inappropriate. Treatment should be discontinued in patients with elevation of liver enzymes on therapy to avoid adaptive liver cell regeneration and potential subsequent tumour formation. Taking a divergent view on the apparent preclinical tumorigenicity of prolonged exposure to micafungin, the Food and Drug Administration did not impose this kind of restriction [92].

\section{RECOMMENDATIONS IN GUIDELINES}

Based on the results of the described clinical trials, an expert group of the Infectious Disease Society of America [67] recommends echinocandins as the preferred treatment in moderately to severely ill non-neutropenic adult patients with suspected or documented candidemia before pathogen species identification. In particular, the IDSA committee recommends the use of anidulafungin, caspofungin or micafungin at an evidence level of A-I. Fluconazole should be restricted to less severely ill patients without recent exposure to an azole antifungal, and those patients without an elevated risk of involvement of C. glabrata or C. krusei (such as cancer patients or elderly patients) [93-95]. For infections with documented involvement of $C$. glabrata, the IDSA panel recommends an echinocandin, whereas fluconazole is preferred for documented C. parapsilosis infections. In terms of prophylaxis, micafungin at $50 \mathrm{mg} / \mathrm{d}$ is considered an option for allogeneic stem cell recipients with neutropenia (evidence level A-I).

On a conference in 2009, European Conference on Infections in Leukemia (ECIL-3) panel issued similar guidance $[76,96]$ : echinocandins or liposomal ampho- tericin $\mathrm{B}$ are recommended with the highest assigned evidence level for treatment of candidemia before species identification in the general patient population and in hematologic patients. Azoles should not be used in patients with previous azole prophylaxis, and fluconazole is restricted to non-severely ill patients. After species identification, echinocandins or liposomal amphotericin B are treatments of choice in patients infected with $C$. albicans, $C$. glabrata, and $C$. krusei. Fluconazole should not be used for C. glabrata and $C$. kruse $i$ infections. There is no guidance provided on preferred treatments for C. parapsilosis infections.

In their guidelines for hematological patients, the Infectious Diseases Working Party (AGIHO) of the German Society of Hematology and Oncology (DGHO) prefers an echinocandin to fluconazole for initial therapy of invasive candidiasis in unstable or neutropenic patients. This recommendation is based on the increasing incidence of infections with Candida spp. with primary resistance or reduced susceptibility to fluconazole and its lower efficacy versus anidulafungin $[55,97]$.

While the optimum treatment duration has not been specifically studied, the guideline panels recommended a duration of therapy for candidemia without obvious metastatic complications of at least 2 weeks after documented eradication of Candida species from the bloodstream plus resolution of symptoms attributable to candidemia plus resolution of neutropenia, if applicable [67, 76, 96, 97].

\section{ROLE OF THE ECHINOCANDINS IN THE MANAGEMENT OF INVASIVE CANDIDA INFECTIONS}

The echinocandins combine a number of features required for the optimization of primary therapy of invasive Candida infections, providing enhanced opportunities of effective and safe treatment over the azole antifungals, particularly fluconazole that is still used as primary therapy in the majority of patients with candidemia (Table 3; Fig. 2) [98].

In contrast to the azoles they are fungicidal against most Candida strains [20], potentially allowing for more rapid clearance of the pathogen from the infection sites. While fungicidality may prove advantageous in severely ill patients, particularly those with severe sepsis, this could not yet be demonstrated in clinical studies since the proportion of critically ill patients in the only available trial of an echinocandin vs. an azole in this indication was below $20 \%$. At least for anidulafungin, a significantly higher success rate in the primary endpoint of a randomized trial versus fluconazole indicates potential therapeutic advantages of echinocandins over azoles in the treatment of candidemia

Table 3. Agents used for the treatment of candidemia as reported in the PATH alliance database [98].

\begin{tabular}{lccccc}
\hline $\mathrm{N}=2019$ patients & Fluconazole & Echinocandins & $\begin{array}{c}\text { Lipid-formulation } \\
\text { amphotericin B }\end{array}$ & $\begin{array}{c}\text { Voriconazole } \\
\text { Amphotericin B } \\
\text { deoxycholate }\end{array}$ \\
\hline Patients treated, \% & 67.7 & 48.9 & 10.0 & 6.7 & 2.2 \\
\hline
\end{tabular}




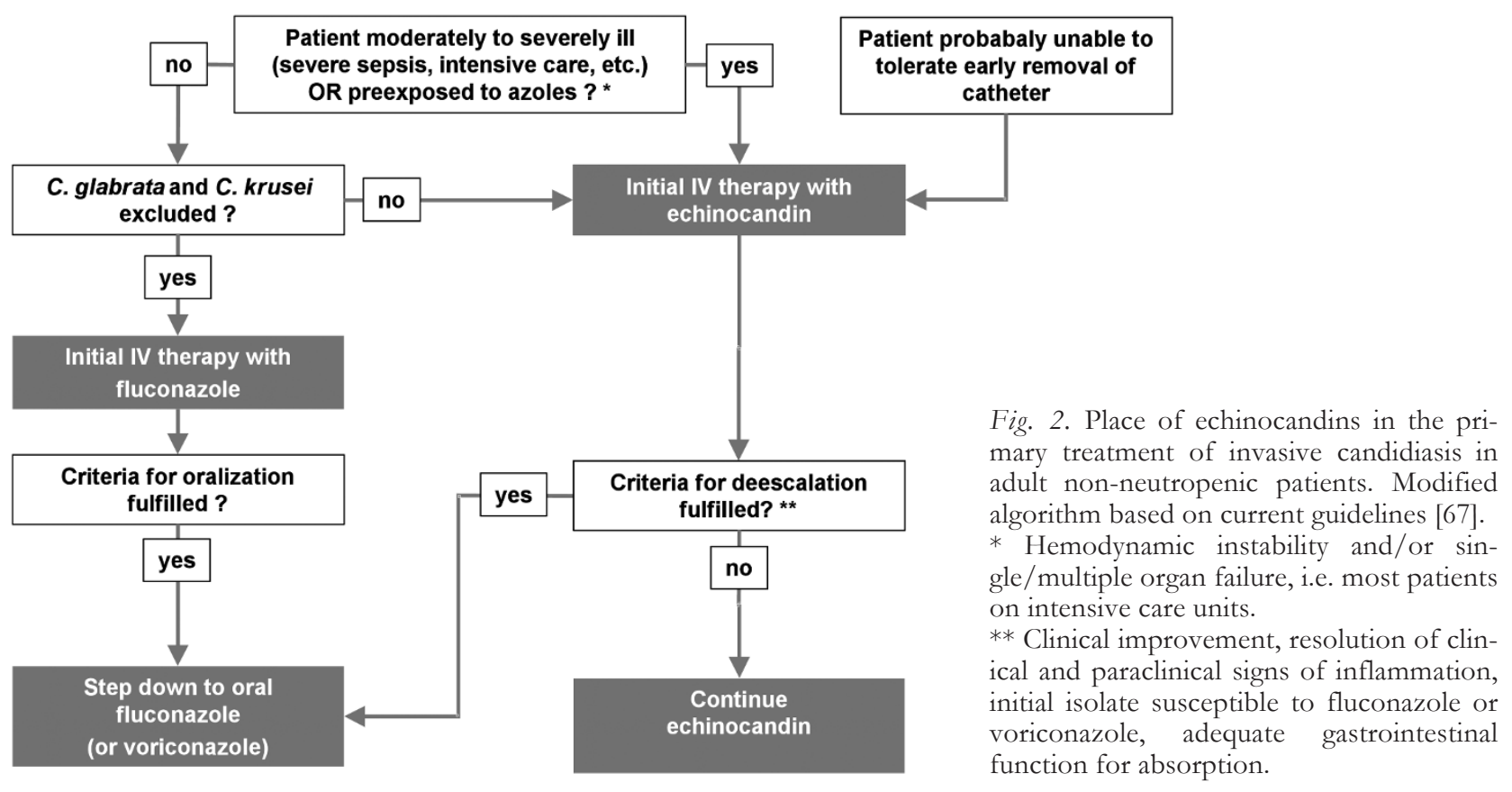

[55]. Also evidence of a shortened median time to blood culture negativity vs. fluconazole appears to point in that direction [59].

Being fully active against C. glabrata and C. krusei, the fungal Achilles' heels of fluconazole, the echinocandins provide an important measure of additional therapeutic reliability in situations where treatment must be started in absence of species identification or even before availability of blood culture results, particularly in elderly patients with high rates of C. glabrata infection [94], and for hematologic and solid cancer patients in whom very high rates of fluconazole-resistant isolates have been described in a recent candidemia survey [95]. Again, this is particularly relevant in patients with severe sepsis or septic shock with a narrow time window of opportunity for initiation of effective therapy to preserve optimum chances of survival $[99,100]$.

Elevated inhibitory concentrations observed in $C$. parapsilosis are causing some concern, but several aspects may alleviate these concerns: (i) a meta-analysis revealed no disadvantage for echinocandins vs. other therapies; [22] (ii) C. parapsilosis is considered as a less virulent compared to e.g. C. albicans and is associated with lower mortality rates [98], (iii) recently proposed resistance breakpoints for C. parapsilosis are several steps higher than for the other clinically relevant $C a n$ dida species [23]; and (iv) current guidelines recommend echinocandins for primary therapy in the absence of species information $[67,76,97]$.

Organ dysfunctions are common in patients developing invasive fungal infections. Being pharmacokinetically independent of renal function, the echinocandins need no dose adjustment in patients with all grades of renal insufficiency or renal replacement therapies, quite different from fluconazole that needs complex dose adjustments in patients with impaired renal function and hemodialysis or hemofiltration [101]. In fact, renal impairment was identified as a significant predictor of inadequate fluconazole dosing [102].
In patients with liver dysfunction, anidulafungin and micafungin may be administered without dose adjustments $[41,47]$. Anidulafungin can be used in patients with severe hepatic insufficiency [41]. The other echinocandins should not be used in this population for lack of data.

The low propensity of echinocandins for drug interactions may allow for substantial simplification of treatment in severely ill patients receiving multiple comedications. Being inhibitors and substrates of hepatic enzymes, the azoles are associated with a multitude of interactions [103] complicating patient management and potentially jeopardizing treatment success. This issue is avoided with anidulafungin and greatly reduced to interactions with few immunosuppressive drugs with the other echinocandins.

Activity against biofilms is another feature that favours the echinocandins over azoles as agents of choice in patients with catheter-associated candidemia particularly in situations where early removal of catheters appears unfeasible. Even in patients whose catheters are removed within the first two days of therapy, echinocandins may suppress fungal burden more effectively during this critical initial treatment phase when the potentially fungus-shedding device is still present.

Prophylactic use of echinocandins currently is an option for selected hematological patients in whom azoles are inappropriate due to tolerability issues or unmanageable drug interactions. Mind that anidulafungin and caspofungin are not licensed for prophylaxis and micafungin is not licensed for prophylaxis of mould infections, a predominant concern in hematological patients [29, 41, 47].

In conclusion, echinocandins provide safe, uncomplicated and highly active therapy for invasive Candida infections with potentially superior efficacy versus fluconazole and better tolerability compared to formulations of amphotericin B, making them the agents of choice for moderately to severely ill patients with invasive candidiasis. 


\section{REFERENCES}

1. Wey SB, Mori M, Pfaller MA, Woolson RF, Wenzel RP (1988) Hospital-acquired candidemia. The attributable mortality and excess length of stay. Arch Intern Med 148:2642-2645.

2. Voss A, le Noble JL, Verduyn Lunel FM, Foudraine NA, Meis JF (1997) Candidemia in intensive care unit patients: risk factors for mortality. Infection 25:8-11.

3. Blot SI, Vandewoude KH, Hoste EA, Colardyn FA (2002) Effects of nosocomial candidemia on outcomes of critically ill patients. Am J Med 113:480-485.

4. Wisplinghoff H, Bischoff T, Tallent SM, Seifert H, Wenzel RP, Edmond MB (2004) Nosocomial bloodstream infections in US hospitals: analysis of 24,179 cases from a prospective nationwide surveillance study. Clin Infect Dis 39:309-317.

5. Gudlaugsson O, Gillespie S, Lee K, Vande Berg J, Hu J, Messer S, Herwaldt L, Pfaller M, Diekema D (2003) Attributable mortality of nosocomial candidemia, revisited. Clin Infect Dis 37: 1172-1177.

6. Zaoutis TE, Argon J, Chu J, Berlin JA, Walsh TJ, Feudtner C (2005) The epidemiology and attributable outcomes of candidemia in adults and children hospitalized in the United States: a propensity analysis. Clin Infect Dis 41:1232-1239.

7. Morrell M, Fraser VJ, Kollef MH (2005) Delaying the empiric treatment of candida bloodstream infection until positive blood culture results are obtained: a potential risk factor for hospital mortality. Antimicrob Agents Chemother 49: 3640-3645.

8. Garey KW, Rege M, Pai MP, Mingo DE, Suda KJ, Turpin RS, Bearden DT (2006) Time to initiation of fluconazole therapy impacts mortality in patients with candidemia: a multi-institutional study. Clin Infect Dis 43:25-31.

9. Vincent JL, Rello J, Marshall J, Silva E, Anzueto A, Martin CD, Moreno R, Lipman J, Gomersall C, Sakr Y, Reinhart K; EPIC II Group of Investigators (2009) International study of the prevalence and outcomes of infection in intensive care units. JAMA 302:2323-2329.

10. Engel C, Brunkhorst FM, Bone HG, Brunkhorst R, Gerlach H, Grond S, Gruendling M, Huhle G, Jaschinski U, John S, Mayer K, Oppert M, Olthoff D, Quintel M, Ragaller M, Rossaint R, Stuber F, Weiler N, Welte T, Bogatsch H, Hartog C, Loeffler M, Reinhart K (2007) Epidemiology of sepsis in Germany: results from a national prospective multicenter study. Intensive Care Med 33:606618.

11. Tortorano AM, Kibbler C, Peman J, Bernhardt H, Klingspor L, Grillot R (2006) Candidaemia in Europe: epidemiology and resistance. Int $\mathrm{J}$ Antimicrob Agents 27:359-366.

12. Guery BP, Arendrup MC, Auzinger G Azoulay E, Borges Sá M, Johnson EM, Müller E, Putensen C, Rotstein C, Sganga G, Venditti M, Zaragoza Crespo R, Kullberg BJ (2009) Management of invasive candidiasis and candidemia in adult non-neutropenic intensive care unit patients: Part I. Epidemiology and diagnosis. Intensive Care Med 35:55-62.

13. Muñoz P, Burillo A, Bouza E (2000) Criteria used when initiating antifungal therapy against Candida spp. in the intensive care unit. Int J Antimicrob Agents 15:83-90.

14. Singh N (2003) Fungal infections in the recipients of solid organ transplantation. Infect Dis Clin North Am 17:113134.

15. Pelz RK, Lipsett PA, Swoboda SM, Diener-West M, Hammond JM, Hendrix CW (2000) The diagnostic value of fungal surveillance cultures in critically ill patients. Surg Infect (Larchmt); 1:273-281.

16. Rotstein C, Bow EJ, Laverdiere M, Ioannou S, Carr D, Moghaddam N (1999). Randomized placebo-controlled trial of fluconazole prophylaxis for neutropenic cancer patients: benefit based on purpose and intensity of cytotoxic therapy. The Canadian Fluconazole Prophylaxis Study Group. Clin Infect Dis 28:331-340.

17. Pagano L, Caira M, Valentini CG, Posteraro B, Fianchi L (2010). Current therapeutic approaches to fungal infections in immunocompromised hematological patients. Blood Rev 24:51-61.

18. Ellis ME, Al-Abdely H, Sandridge A, Greer W, Ventura W (2001) Fungal endocarditis: evidence in the world literature, 1965-1995. Clin Infect Dis 32:50-62.

19. Denning DW (2003). Echinocandin antifungal drugs. Lancet 362:1142-1151.

20. Pound MW, Townsend ML, Drew RH. Echinocandin pharmacodynamics: review and clinical implications. J Antimicrob Chemother. 2010 Jun;65(6):1108-18.

21. Pfaller MA, Boyken L, Hollis RJ, Kroeger J, Messer SA, Tendolkar S, Jones RN, Turnidge J, Diekema DJ. Wildtype MIC distributions and epidemiological cutoff values for the echinocandins and Candida spp. J Clin Microbiol. 2010 Jan;48(1):52-6.

22. Kale-Pradhan PB, Morgan G, Wilhelm SM, Johnson LB (2010) Comparative efficacy of echinocandins and nonechinocandins for the treatment of Candida parapsilosis Infections: a meta-analysis. Pharmacotherapy 30:12071213.

23. Pfaller M, Diekema D, Andes D, et al (2010) Clinical Breakpoints for the Echinocandins and Candida, Revisited. Poster M-369; 50th ICAAC, Boston, MA, USA.

24. Sun HY, Singh N (2010) Characterisation of breakthrough invasive mycoses in echinocandin recipients: an evidencebased review. Int J Antimicrob Agents 35:211-218.

25. Perlin DS (2007) Resistance to echinocandin-class antifungal drugs. Drug Resist Updat 10:121-130.

26. Cota J, Carden M, Graybill JR, Najvar LK, Burgess DS, Wiederhold NP (2006) In vitro pharmacodynamics of anidulafungin and caspofungin against Candida glabrata isolates, including strains with decreased caspofungin susceptibility. Antimicrob Agents Chemother 50(11):39263928.

27. Edlind T, Healey K, Jphnson M, Katiyar S. et al. (2010) Candida glabrata CRS-MIS Mutants: Caspofungin Reduced Susceptibility but Micafungin Increased Susceptibility. Poster M-393; 50th ICAAC, Boston, MA, USA.

28. Migoya EM, Mistry GC, Stone JA, Comisar W, Sun P, Norcross A, Bi S, Winchell GA, Ghosh K, Uemera N, Deutsch PJ, Wagner JA (2011) Safety and pharmacokinetics of higher doses of caspofungin in healthy adult participants. J Clin Pharmacol 51:202-211.

29. European Medicines Agency (2009) Cancidas ${ }^{\circledR}$ summary of product characteristics.

30. Niwa T, Yokota Y, Tokunaga A, Yamato Y, Kagayama A, Fujiwara T, Hatakeyama J, Anezaki M, Ohtsuka Y, Takagi A (2004) Tissue distribution after intravenous dosing of micafungin, an antifungal drug, to rats. Biol Pharm Bull 27:1154-1156.

31. Hajdu R, Thompson R, Sundelof JG, Pelak BA, Bouffard FA, Dropinski JF, Kropp H (1997) Preliminary animal pharmacokinetics of the parenteral antifungal agent MK0991 (L-743,872). Antimicrob Agents Chemother 41:2339-2344.

32. Damle B, Stogniew M, Dowell J (2008) Pharmacokinetics and tissue distribution of anidulafungin in rats. Antimicrob Agents Chemother 52:2673-2676.

33. Deresinski SC, Stevens DA (2003) Caspofungin. Clin Infect Dis 36:1445-1457.

34. Chandrasekar PH, Sobel JD (2006) Micafungin: a new echinocandin. Clin Infect Dis 42:1171-1178.

35. Vazquez JA, Sobel JD (2006) Anidulafungin: a novel echinocandin. Clin Infect Dis 43:215-222.

36. Damle BD, Dowell JA, Walsky RL, Weber GL, Stogniew M, Inskeep PB (2009). In vitro and in vivo studies to characterize the clearance mechanism and potential cytochrome P450 interactions of anidulafungin. Antimicrob Agents Chemother 53:1149-1156. 
37. Eschenauer G, Depestel DD, Carver PL (2007) Comparison of echinocandin antifungals. Ther Clin Risk Manag 3:71-97.

38. Krause DS, Reinhardt J, Vazquez JA, Reboli A, Goldstein BP, Wible M, Henkel T; Anidulafungin Invasive Candidiasis Study Group (2004). Phase 2, randomized, dose-ranging study evaluating the safety and efficacy of anidulafungin in invasive candidiasis and candidemia. Antimicrob Agents Chemother 48:2021-2024.

39. Dowell JA, Knebel W, Ludden T, Stogniew M, Krause D, Henkel T (2004) Population pharmacokinetic analysis of anidulafungin, an echinocandin antifungal. J Clin Pharmacol 44:590-598.

40. Dowell JA, Stogniew M, Krause D, Damle B (2007) Anidulafungin does not require dosage adjustment in subjects with varying degrees of hepatic or renal impairment. J Clin Pharmacol 47:461-470.

41. European Medicines Agency (2010) Ecalta ${ }^{\circledR}$ summary of product characteristics.

42. Stone JA, Migoya EM, Hickey L, Winchell GA, Deutsch PJ, Ghosh K, Freeman A, Bi S, Desai R, Dilzer SC, Lasseter KC, Kraft WK, Greenberg H, Waldman SA (2004) Potential for interactions between caspofungin and nelfinavir or rifampin. Antimicrob Agents Chemother 48:4306-4314.

43. Betts RF, Nucci M, Talwar D, Gareca M, Queiroz-Telles F, Bedimo RJ, Herbrecht R, Ruiz-Palacios G, Young JA, Baddley JW, Strohmaier KM, Tucker KA, Taylor AF, Kartsonis NA; Caspofungin High-Dose Study Group (2009) A Multicenter, double-blind trial of a high-dose caspofungin treatment regimen versus a standard caspofungin treatment regimen for adult patients with invasive candidiasis. Clin Infect Dis 48:1676-1684.

44. Mistry GC, Migoya E, Deutsch PJ, Winchell G, Hesney M, Li S, Bi S, Dilzer S, Lasseter KC, Stone JA (2007) Single- and multiple-dose administration of caspofungin in patients with hepatic insufficiency: implications for safety and dosing recommendations. J Clin Pharmacol 47:951961.

45. Pappas PG, Rotstein CM, Betts RF, Nucci M, Talwar D, De Waele JJ, Vazquez JA, Dupont BF, Horn DL, Ostrosky-Zeichner L, Reboli AC, Suh B, Digumarti R, Wu C, Kovanda LL, Arnold LJ, Buell DN (2007). Micafungin versus caspofungin for treatment of candidemia and other forms of invasive candidiasis. Clin Infect Dis 45:883-893.

46. van Burik JA, Ratanatharathorn V, Stepan DE, Miller CB, Lipton JH, Vesole DH, Bunin N, Wall DA, Hiemenz JW, Satoi Y, Lee JM, Walsh TJ; National Institute of Allergy and Infectious Diseases Mycoses Study Group (2004) Micafungin versus fluconazole for prophylaxis against invasive fungal infections during neutropenia in patients undergoing hematopoietic stem cell transplantation. Clin Infect Dis 39:1407-1416.

47. European Medicines Agency (2010) Mycamine ${ }^{\circledR}$ summary of product characteristics.

48. Gubbins PO, Heldenbrand S (2010) Clinically relevant drug interactions of current antifungal agents. Mycoses 53:95-113.

49. Finch RG, Greenwood D, Norrby SR (2003) Antibiotic and chemotherapy: anti-infective agents and their use in therapy. 8th edition; Elsevier Publishers.

50. Dowell JA, Stogniew M, Krause D, Henkel T, Weston IE (2005) Assessment of the safety and pharmacokinetics of anidulafungin when administered with cyclosporine. J Clin Pharmacol 45:227-233.

51. Dowell JA, Stogniew M, Krause D, Henkel T, Damle B (2007) Lack of pharmacokinetic interaction between anidulafungin and tacrolimus. J Clin Pharmacol 47:305-314.

52. Dowell JA, Schranz J, Baruch A, Foster G (2005). Safety and pharmacokinetics of coadministered voriconazole and anidulafungin. J Clin Pharmacol 45:1373-1382.

53. Marr KA, Hachem R, Papanicolaou G, Somani J, Arduino JM, Lipka CJ, Ngai AL, Kartsonis N, Chodakewitz J,
Sable C (2004). Retrospective study of the hepatic safety profile of patients concomitantly treated with caspofungin and cyclosporin A. Transpl Infect Dis 6:110-116.

54. Sable CA, Nguyen BY, Chodakewitz JA, DiNubile MJ (2002) Safety and tolerability of caspofungin acetate in the treatment of fungal infections. Transpl Infect Dis 4:25-30.

55. Reboli AC, Rotstein C, Pappas PG, Chapman SW, Kett DH, Kumar D, Betts R, Wible M, Goldstein BP, Schranz J, Krause DS, Walsh TJ; Anidulafungin Study Group (2007) Anidulafungin versus fluconazole for invasive candidiasis. N Engl J Med 356:2472-2482.

56. Mora-Duarte J, Betts R, Rotstein C, Colombo AL, Thompson-Moya L, Smietana J, Lupinacci R, Sable C, Kartsonis N, Perfect J; Caspofungin Invasive Candidiasis Study Group (2002) Comparison of caspofungin and amphotericin B for invasive candidiasis. $\mathrm{N}$ Engl J Med 347:2020-2029.

57. Kuse ER, Chetchotisakd P, da Cunha CA, Ruhnke M, Barrios C, Raghunadharao D, Sekhon JS, Freire A, Ramasubramanian V, Demeyer I, Nucci M, Leelarasamee A, Jacobs F, Decruyenaere J, Pittet D, Ullmann AJ, OstroskyZeichner L, Lortholary O, Koblinger S, Diekmann-Berndt $\mathrm{H}$, Cornely OA; Micafungin Invasive Candidiasis Working Group (2007) Micafungin versus liposomal amphotericin $\mathrm{B}$ for candidaemia and invasive candidosis: a phase III randomised double-blind trial. Lancet 369:1519-1527.

58. Rex JH, Bennett JE, Sugar AM, Pappas PG, van der Horst CM, Edwards JE, Washburn RG, Scheld WM, Karchmer AW, Dine AP, et al. (1994) A randomized trial comparing fluconazole with amphotericin B for the treatment of candidemia in patients without neutropenia. Candidemia Study Group and the National Institute. N Engl J Med 331:1325-1330.

59. Pfizer Inc. (2010); data on file.

60. Kett DH, Shorr AF, Reboli AC, Reisman AL, Biswass P, Schlamm H (2010) Anidulafungin compared with fluconazole therapy in critically ill patients. Critical Care 14 (Suppl 1):P72.

61. Paiva JA, Ruhnke M, Meersseman W, Pachl J, Grigoras I, Sganga G, Menichetti F, Montravers P, Auzinger G, Dimopoulos G, Borges Sá M, Miller P, Marček T, Kantecki M (2010) Anidulafungin for treatment of candidemia/invasive candidiasis in selected intensive care unit populations. Crit Care Med 38:12 (Suppl.):Abstract 297.

62. Cornely OA, Lasso M, Betts R, Klimko N, Vazquez J, Dobb G, Velez J, Williams-Diaz A, Lipka J, Taylor A, Sable C, Kartsonis N (2007) Caspofungin for the treatment of less common forms of invasive candidiasis. J Antimicrob Chemother 60:363-369.

63. Colombo AL, Ngai AL, Bourque M, Bradshaw SK, Strohmaier KM, Taylor AF, Lupinacci RJ, Kartsonis NA (2010) Caspofungin use in patients with invasive candidiasis caused by common non-albicans Candida species: review of the caspofungin database. Antimicrob Agents Chemother 54:1864-1871.

64. Betts R, Glasmacher A, Maertens J, Maschmeyer G, Vazquez JA, Teppler H, Taylor A, Lupinacci R, Sable C, Kartsonis N (2006) Efficacy of caspofungin against invasive Candida or invasive Aspergillus infections in neutropenic patients. Cancer 106:466-473.

65. Lichtenstern C, Nguyen TH, Schemmer P, Hoppe-Tichy T, Weigand MA (2008) Efficacy of caspofungin in invasive candidiasis and candidemia--de-escalation strategy. Mycoses 51 Suppl 1:35-46.

66. Dunyach C, Drakulovski P, Bertout S, Jouvert S, Reynes J, Mallié M (2010) Fungicidalactivity and morphological alterations of Candida albicans induced by echinocandins: study of strains with reduced caspofungin susceptibility. Mycoses [Epub ahead of print].

67. Pappas PG, Kauffman CA, Andes D, Benjamin DK Jr, Calandra TF, Edwards JE Jr, Filler SG, Fisher JF, Kullberg BJ, Ostrosky-Zeichner L, Reboli AC, Rex JH, Walsh TJ, Sobel JD; Infectious Diseases Society of America 
(2009) Clinical practice guidelines for the management of candidiasis: 2009 update by the Infectious Diseases Society of America. Clin Infect Dis 48:503-535.

68. Katragkou A, Chatzimoschou A, Simitsopoulou M, Dalakiouridou M, Diza-Mataftsi E, Tsantali C, Roilides E. Differential activities of newer antifungal agents against Candida albicans and Candida parapsilosis biofilms (2008) Antimicrob Agents Chemother 52:357-360.

69. Kuhn DM, George T, Chandra J, Mukherjee PK, Ghannoum MA (2002) Antifungal susceptibility of Candida biofilms: unique efficacy of amphotericin $\mathrm{B}$ lipid formulations and echinocandins. Antimicrob Agents Chemother 46:1773-1780.

70. Kucharíková S, Tournu H, Holtappels M, Van Dijck P, Lagrou K (2010) In vivo efficacy of anidulafungin against mature Candida albicans biofilms in a novel rat model of catheter-associated Candidiasis. Antimicrob Agents Chemother 54:4474-4475.

71. Jacobson MJ, Piper KE, Nguyen G, Steckelberg JM, Patel $\mathrm{R}$ (2008) In vitro activity of anidulafungin against Candida albicans biofilms. Antimicrob Agents Chemother 52:22422243.

72. Shuford JA, Piper KE, Steckelberg JM, Patel R (2007) In vitro biofilm characterization and activity of antifungal agents alone and in combination against sessile and planktonic clinical Candida albicans isolates. Diagn Microbiol Infect Dis 57:277-281.

73. Katragkou A, Chatzimoschou A, Simitsopoulou M, Georgiadou E, Roilides E (2010) Additive antifungal activity of anidulafungin and human neutrophils against Candida parapsilosis biofilms. J Antimicrob Chemother [Epub ahead of print].

74. Katragkou A, Kruhlak MJ, Simitsopoulou M, Chatzimoschou A, Taparkou A, Cotten CJ, Paliogianni F, DizaMataftsi E, Tsantali C, Walsh TJ, Roilides E (2010) Interactions between human phagocytes and Candida albicans biofilms alone and in combination with antifungal agents. J Infect Dis 201:1941-1949.

75. Nucci M, Anaissie E, Betts RF, Dupont BF, Wu C, Buell DN, Kovanda L, Lortholary O (2010) Early removal of central venous catheter in patients with candidemia does not improve outcome: analysis of 842 patients from 2 randomized clinical trials. Clin Infect Dis 51:295-303.

76. Herbrecht R, Flückinger U, Gachot B, Ribaud P, Thiebaut A, Cordonnier C (2009) Antifungal therapy in leukemia patients : 2009 Update of the ECIL1 and ECIL 2 guidelines. www.ichs.org/Resources/Documents/ECIL 3 Antifungal therapy Update 2009.pdf

77. Zaoutis TE, Jafri HS, Huang LM, Locatelli F, Barzilai A, Ebell W, Steinbach WJ, Bradley J, Lieberman JM, Hsiao CC, Seibel N, Laws HJ, Gamba M, Petrecz M, Taylor AF, Strohmaier KM, Chow JW, Kartsonis NA, Ngai AL (2009) A prospective, multicenter study of caspofungin for the treatment of documented Candida or Aspergillus infections in pediatric patients. Pediatrics 123:877-884.

78. Queiroz-Telles F, Berezin E, Leverger G, Freire A, van der Vyver A, Chotpitayasunondh T, Konja J, DiekmannBerndt H, Koblinger S, Groll AH, Arrieta A; Micafungin Invasive Candidiasis Study Group (2008) Micafungin versus liposomal amphotericin B for pediatric patients with invasive candidiasis: substudy of a randomized doubleblind trial. Pediatr Infect Dis J 27:820-826.

79. Benjamin DK Jr, Driscoll T, Seibel NL, Gonzalez CE, Roden MM, Kilaru R, Clark K, Dowell JA, Schranz J, Walsh TJ. Safety and pharmacokinetics of intravenous anidulafungin in children with neutropenia at high risk for invasive fungal infections. Antimicrob Agents Chemother. 2006 Feb;50(2):632-8.

80. www.clinicaltrials.gov; trial identifier NCT00761267.

81. Almyroudis NG, Segal BH (2010) Antifungal prophylaxis and therapy in patients with hematological malignancies and hematopoietic stem cell transplant recipients. Expert Rev Anti Infect Ther 8:1451-1466.
82. Playford EG, Lipman J, Sorrell TC (2010) Prophylaxis, empirical and preemptive treatment of invasive candidiasis. Curr Opin Crit Care 16:470-474.

83. Mattiuzzi GN, Alvarado G, Giles FJ, Ostrosky-Zeichner L, Cortes J, O'brien S, Verstovsek S, Faderl S, Zhou X, Raad II, Bekele BN, Leitz GJ, Lopez-Roman I, Estey EH (2006). Open-label, randomized comparison of itraconazole versus caspofungin for prophylaxis in patients with hematologic malignancies. Antimicrob Agents Chemother 50:143-147.

84. Chou LS, Lewis RE, Ippoliti C, Champlin RE, Kontoyiannis DP (2007) Caspofungin as primary antifungal prophylaxis in stem cell transplant recipients. Pharmacotherapy 27:1644-1650.

85. Fortún J, Martín-Dávila P, Montejo M, Muñoz P, Cisneros JM, Ramos A, Aragón C, Blanes M, San Juan R, Gavaldá J, Llinares P; GESITRA Study Group (2009) Prophylaxis with caspofungin for invasive fungal infections in high-risk liver transplant recipients. Transplantation 87:424-435.

86. Cappelletty D, Eiselstein-McKitrick K (2007) The echinocandins. Pharmacotherapy; 27:369-388.

87. Food and Drug Administration, USA (2010) Prescription Information Eraxis ${ }^{\circledR}$.

88. Food and Drug Administration, USA (2010) Prescription Information Cancidas ${ }^{\circledR}$.

89. Food and Drug Administration, USA (2010) Prescription Information Mycamine ${ }^{\circledR}$.

90. Reboli AC, Rotstein C, Pappas PG, Chapman SW, Kett DH, Kumar D, Betts R, Wible M, Goldstein BP, Schranz J, Krause DS, Walsh TJ; Anidulafungin Study Group (2007) Anidulafungin versus fluconazole for invasive candidiasis. N Engl J Med 356:2472-2482. Supplement available at the journal website (www.nejm.org).

91. European Medicines Agency (2010) Mycamine ${ }^{\circledR}$ summary of product characteristics.

92. Food and Drug Administration (2009) Mycamine ${ }^{\circledR}$ summary of product characteristics.

93. Pfaller MA, Castanheira M, Messer SA, Moet GJ, Jones RN (2010) Variation in Candida spp. distribution and antifungal resistance rates among bloodstream infection isolates by patient age: report from the SENTRY Antimicrobial Surveillance Program (2008-2009). Diagn Microbiol Infect Dis 2010 68:278-283.

94. Sandven P, Bevanger L, Digranes A, Haukland HH, Mannsåker T, Gaustad P; Norwegian Yeast Study Group (2006) Candidemia in Norway (1991 to 2003): results from a nationwide study. J Clin Microbiol 44:19771981.

95. Slavin MA, Sorrell TC, Marriott D, Thursky KA, Nguyen Q, Ellis DH, Morrissey CO, Chen SC; Australian Candidemia Study, Australasian Society for Infectious Diseases (2010). Candidaemia in adult cancer patients: risks for fluconazole-resistant isolates and death. J Antimicrob Chemother 65:1042-1051.

96. Maertens J, Marchetti O, Herbrecht R, Cornely OA, Flückiger U, Frêre P, Gachot B, Heinz WJ, Lass-Flörl C, Ribaud P, Thiebaut A, Cordonnier C (2010) European guidelines for antifungal management in leukemia and hematopoietic stem cell transplant recipients: summary of the ECIL 3-2009 Update. Bone Marrow Transplant [Epub ahead of print].

97. Infectious Diseases Working Party (AGIHO) of the German Society of Hematology and Oncology (DGHO), Böhme A, Ruhnke M, Buchheidt D, Cornely OA, Einsele H,Enzensberger R, Hebart H, Heinz W, Junghanss C, Karthaus M, Krüger W, Krug U, Kubin T, Penack O, Reichert D, Reuter S, Silling G, Südhoff T, Ullmann AJ, Maschmeyer G (2009) Treatment of invasive fungal infections in cancer patients--recommendations of the Infectious Diseases Working Party (AGIHO) of the German Society of Hematology and Oncology (DGHO). Ann Hematol 88:97-110. 
98. Horn DL, Neofytos D, Anaissie EJ, Fishman JA, Steinbach WJ, Olyaei AJ, Marr KA, Pfaller MA, Chang CH, Webster KM (2009) Epidemiology and outcomes of candidemia in 2019 patients: data from the prospective antifungal therapy alliance registry. Clin Infect Dis 48:16951703.

99. Kumar A, Skrobik I, Guzman J, Lapinsky S, Laupland K, CATSS Database Research Group (2007) The high mortality of Candida septic shock is explained by excessive delays in initiation of antifungal therapy. Poster K-2174; 47th ICAAC, Chicago, IL, USA.

100. Taur Y, Cohen N, Dubnow S, Paskovaty A, Seo SK (2010) Effect of antifungal therapy timing on mortality in cancer patients with candidemia. Antimicrob Agents Chemother 54:184-190.

101. European Medicines Agency (2010) Diflucan ${ }^{\circledR}$ summary of product characteristics.

102. Garey KW, Pai MP, Suda KJ, Turpin RS, Rege MD, Mingo DE, Bearden DT (2007) Inadequacy of fluconazole dosing in patients with candidemia based on Infectious Diseases Society of America (IDSA) guidelines. Pharmacoepidemiol Drug Saf 16:919-927.
103. Nivoix Y, Ubeaud-Sequier G, Engel P, Levêque D, Herbrecht R (2009) Drug-drug interactions of triazole antifungal agents in multimorbid patients and implications for patient care. Curr Drug Metab 10:395-409.

Received: February 10, 2011 / Accepted: February 16, 2011

Address for correspondence:

Andreas Glöckner

BDH Klinik Greifswald GmbH

Karl-Liebknecht-Ring 26A

D-17491 Greifswald

Germany

Phone: +49-(0)3834-871-231

Fax: +49-(0)3834-871-226

Email: a.gloeckner@bdh-klinik-greifswald.de 